\title{
High Resolution Cryo-Electron Microscopy of Virus Capsids at Liquid-Helium Temperature
}

\author{
N. Cheng*, J. F. Conway**, D. C. Winkler*, R. L. Duda ***, A. C. Steven* \\ * Laboratory of Structural Biology, NIAMS-NIH, Bethesda, MD 20892, USA \\ ** Institut de Biologie Structurale CEA-CNRS-UJF, 38027 Grenoble, France \\ *** Department of Biological Sciences, University of Pittsburgh, Pittsburgh, PA 15260, USA
}

A quantum advance in the resolution of 3-D reconstructions of free-standing macromolecules ("single particles") to the sub-1 nm level was achieved in 1997, through exploitation of microscopes with field-emission gun illumination systems and effective computational methods for correcting for the effects of the phase contrast transfer function (CTF) and for optimizing the determination of alignment and orientation parameters [1,2]. Since then, reconstructions in the resolution range of $0.7 \mathrm{~nm}$ to $\sim 1.2 \mathrm{~nm}$ have appeared with increasing frequency, not only for icosahedral virus capsids but also for complexes with lower symmetry. The achievement of resolutions below $0.5 \mathrm{~nm}$ with helical filaments [3] and tubular crystals [4] suggests that comparable results should be possible for at least some "single particles". Such progress is likely to depend on further improvement of both the primary data (micrographs) and the methods of image analysis.

This presentation makes a progress report on a long-term project to optimize the quality of our cryoelectron micrographs recorded on a FEG-equipped electron microscope operating at $300 \mathrm{keV}$ (FEI Polara), for specimens cooled by liquid helium. As test specimen, we have used Prohead I capsids of bacteriophage HK97, $45 \mathrm{~nm}$ in diameter and $16 \mathrm{MDa}$ in mass, which can be obtained in highly uniform preparations. Specimens were vitrified over holey carbon films that had been baked in an electron beam to improve conductivity [4], and were maintained in the microscope at temperatures of $9-10^{\circ} \mathrm{K}$. Data were recorded at 59,000 x magnification and doses of $\sim 1900$ to 2200 electrons $/ \mathrm{nm}^{2}$ on SO163 cut film in holders whose backs had been excised to prevent back-scatter. They were digitized on a Phodis scanner at $7 \mu \mathrm{m}$, and evaluated in terms of the radial limit of "Thon fringes" in diffraction patterns calculated from 64 areas of 1024 x 1024 pixels. Our best results are shown in Figure 1. In micrograph (a), recorded at $1.1 \mu \mathrm{m}$ defocus (first CTF zero at $(1.5 \mathrm{~nm})^{-1}$ ), the Thon fringes extend to $(0.36 \mathrm{~nm})^{-1}$. In micrograph $(\mathrm{b})$, recorded at $3.4 \mu \mathrm{m}$ defocus (first CTF zero at $(2.5$ $\left.\mathrm{nm})^{-1}\right)$, they extend to $(0.49 \mathrm{~nm})^{-1}[5]$.

\section{References}

[1] J. F. Conway et al., Nature 386 (1997) 91.

[2] B. Boettcher et al., Nature 396 (1997) 88.

[3] K. Yonekura et al., Nature 42 (2003) 643.

[4] A. Miyazawa et al., J Mol Biol. 288(1999) 765.

[5] We thank Dr. H. Zhou for discussion, Dr. B. Heymann for advice on computing, Mr. G. Melvin for adapting film holders, Mr. B. Firek for preparing capsids, and Mrs. L. You for assistance. 

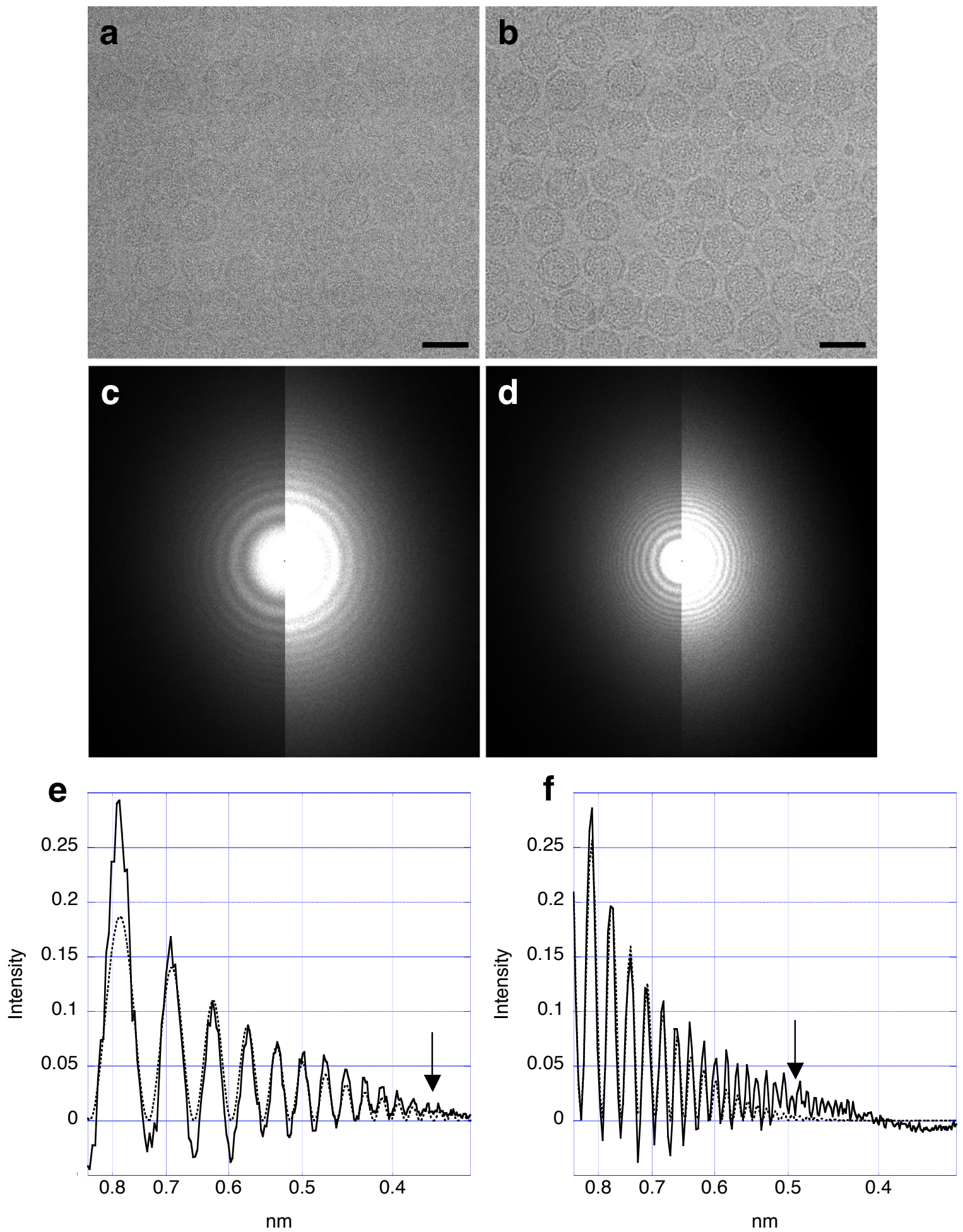

Figure 1. Micrographs of HK97 recorded at $1.1 \mu \mathrm{m}$ (a) and $3.4 \mu \mathrm{m}$ (b) defocus. Scale bars are $50 \mathrm{~nm}$. (c) and (d) are the power spectra of (a) and (b), respectively, calculated from 64 areas of 1024 x 1024 pixels. Background-subtracted radial power spectra are shown by solid lines in (e) and (f), with arrows indicating the extent of the "Thon fringes." Dotted lines indicate the calculated CTF. 